\title{
ECONOMICS OF THICK AND THIN FILM HYBRID PRODUCTION IN EUROPE
}

\author{
D. BOSWELL \\ Film Circuit Product Group, ITT Components Group Europe, Paignton, Devon, U.K. \\ D. S. CAMPBELL \\ Department of Electronic and Electrical Engineering, University of Technology, \\ Loughborough, LE11 3TU, U.K.
}

(Received June 28, 1977)

\begin{abstract}
This paper is a report of a discussion panel held at the First European Conference on Hybrid Microelectronics, concerning the Economics of Hybrid Production. Figures were presented at the discussion showing the sizes of the markets in the U.S.A. and in Europe, demonstrating the percentage of total hybrid markets associated with thin film technology and also the percentage of the market associated with hermetically packaged circuits. It was shown that the growth rate in hybrid technology is similar to that predicted for integrated circuits, a result which can be explained in terms of the interconnection facility which hybrid technology offers.
\end{abstract}

\section{INTRODUCTION}

An interesting feature of the First European Conference on Hybrid Microelectronics at Bad Homburg was the two panel discussions which were held before dinner on the two evenings available. One of these was devoted to chip mounting and interconnection and was chaired by Dr. Hans Delfs from Rhode and Schwarz, Munich. The other was concerned with the Economics of Hybrid Circuits, chaired by Mr. David Boswell of I.T.T., Paignton, U.K. These notes are related to the second panel discussion and represent an attempt to summarise some of the thoughts put forward.

The panel consisted of the following members, representing the various countries:-

$\begin{array}{ll}\text { Mr. D. Boswell } & \text { Chairman. U.K. } \\ \text { Herr P. Bauer } & \text { Germany } \\ \text { Dr. B. Dreyfus-Alain } & \text { France } \\ \text { Prof. F. Forlani } & \text { Italy } \\ \text { L. Larsen } & \text { Scandinavia } \\ \text { Prof. P. L. Kirby } & \text { U.K. } \\ \text { G. Waite } & \text { U.S.A. }\end{array}$

The aim of the discussion was firstly to establish a list of market values for 1976 and predicted figures for 1977 and 1978 and secondly, each member of the Panel was asked to speak to these figures in terms of his own country.

\section{DEFINITION}

Before arriving at an analysis of the market situation, it was realised that it was important to define the meaning of the term "Hybrid Circuit". Prof. Kirby was able to provide such a definition. ${ }^{1}$ As he pointed out, the subject is, by its nature, very diverse and includes a wide range of products varying from large volume production of relatively low cost assemblies to complex, high cost modules sold in small quantities.

It is possible to divide the various products involved into eight categories as follows:-

1) Passive resistor networks.

Tolerances not better than $1 \%$ and involving a simple (e.g. conformal) protection. These usually involve thick film cermet materials.

2) High accuracy passive resistor networks. Involving tolerances down to $10 \mathrm{ppm}$ with low T.C.R. and high stability. These are usually constructed using thin film deposited components.

3) Hybrid assemblies involving simple conductor/ resistor film circuits to which are attached capacitors and/or pre-packaged active devices of simple to medium complexity. The assembly will be conformally coated or encapsulated in a plastic case and usually involves thick film materials.

4) More advanced film circuits involving fine line 
conductors and/or higher accuracy film resistors and added components. In many cases these will involve un-encapsulated i.c. chips and wire bonds (involving say, no more than 50 wire loops). Functional adjustment may be required and these hybrids will usually be in thick films, especially if multilayers are involved, and in some cases will involve hermetic packaging. However, in some cases there is an established preference for the use of thin films.

5) Specialised applications which call for performance parameters not readily achieved from 4) above including, for example, active filters, high accuracy digitisers and other convertors. The performance requirements may require the incorporation of components of a specialised nature such as high accuracy close tolerance resistors (or networks). Functional adjustment and sophisticated test equipment will almost certainly be required. Such assemblies may involve a combination of thick and thin film technology, possibly with multiple substrates in a given package.

6) High definition thick film circuits consisting essentially of fine line conductors (possibly in a multilayer format), to which are added a number of complex i.c. chips including 1.s.i. components. In this case the thick film elements are utilised mainly to provide an interconnection medium (and to form the basis of a suitable packaging system) for a multi-chip 1.s.i. assembly. These assemblies may frequently be characterised by involving a large number of wire loops (e.g. $>50$ and in some cases $>200$ ).

7) Microwave circuits involving geometrically accurate conductor patterns on high quality ceramic substrates. At one time these circuits utilised thin film techniques but thick films are now also frequently used.

8) Opto-electronic assemblies including display assemblies in which conductive films, some of a transparent nature, are utilised in packages with transparent windows.

In addition, thick film technology in particular is used for certain types of both general purpose potentiometers (wattage dissipation $<7 \mathrm{~W}$ and reproducibility to $>5 \%$ ) and precision potentiometers (reproducible to $<0.5 \%$ ). The majority of the general purpose potentiometers are designed to be panel mounted or as trimmers, often being designed to fit directly on to printed circuit boards. Values up to $5 \mathrm{M} \Omega$ are possible with general purpose potentiometers and $1 \mathrm{M} \Omega$ for precision devices.

These eight categories and the additional one of potentiometers are often grouped to give three main areas, namely:-

Networks - including Categories 1 and 2 .

Multichip Hybrids - including Categories 5 to 8

Potentiometers

\section{U.S. SITUATION}

The first speaker was Gilbert Waite, whose paper on the "Economic Progress of Thick Film Hybrids in the U.S.A." was published in the Proceedings of the Conference. ${ }^{2}$ In summary, he made the following points:

1) It is difficult to establish the exact sales figures for hybrids because so much of the production is for internal use; this is particularly true for very large companies such as I.B.M. and Delco and even if one does establish the percentage of production for internal, i.e. captive markets, it is difficult to put an open market value on the production. These comments apply particularly to resistor networks and multichip hybrids. The situation with regard to potentiometers is easier to analyse. Thus it must be realised that all market figures must be interpreted with a wide degree of tolerance.

2) The military market accounts for a very large percentage of the sales in the U.S.A. and figures taken in this respect are that $70 \%$ of total sales go to the military market and $30 \%$ go to commercial requirements.

3) Using these considerations, the following figures have been derived for the year 1977:-

Networks $60 \mathrm{M} \$$

Potentiometers $100 \mathrm{M} \$$

Multichip Hybrids

(These have been estimated from the

known military contracts in existence at present and assume a growth rate from $138 \mathrm{M} \$$ to $168 \mathrm{M} \$$ in 1977 together with $72 \mathrm{M} \$$ of commercial work).

TOTAL $400 \mathrm{M} \$$

Assuming a 3:1 ratio for the captive to non-captive (i.e. commercial) markets, this gives a total in the U.S.A. for resistor networks and multichip hybrids of $1.2 \mathrm{~B} \$$ and if potentiometers are included, a total of 1.3B\$. The potentiometer market is assumed to be only commercial. Figure 1 shows the distribution for the non-captive U.S. market. 


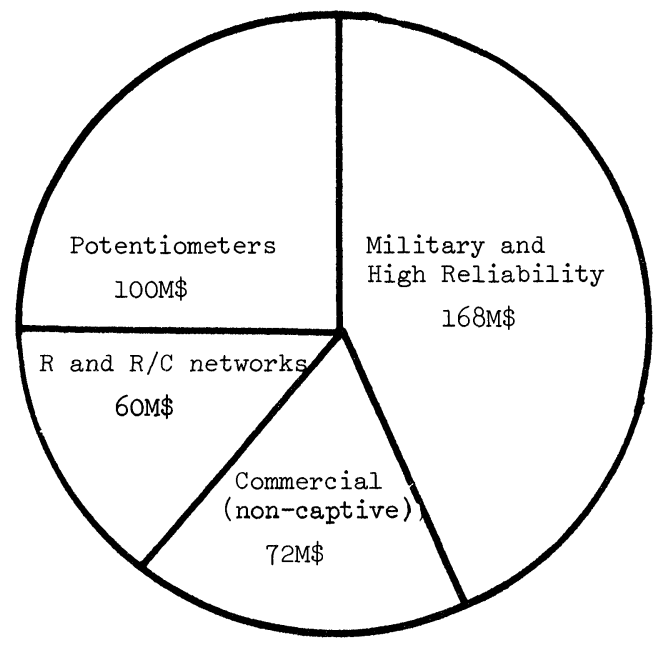

FIGURE 1 Estimated non-captive market in the U.S. for hybrid circuits production in 1977 (after Waite, Ref. 1).

4) The growth picture of the last 12 years shows that the multichip hybrid market has risen from $45 \mathrm{M} \$$ in 1965 to $240 \mathrm{M} \$$ in 1977 . These figures are given graphically in Figure 2. The feature of interest is that the economic recession which has occurred recently does not seem to have made much difference to the general trend, so that one can expect a $12 \%$ $15 \%$ average growth rate hybrid microelectronics for several years to come.

Forecasts for 1977 have appeared at the beginning of 1977 and these figures may be compared with those suggested by Waite. Electronics ${ }^{3}$ gives. figures for non-captive markets with respect to a large variety of different components and the relevant figures are:-

Resistive networks (thin and thick film) $52.2 \mathrm{M} \$$ Potentiometers (non wirewound) $122.0 \mathrm{M \$}$ Multi-component and hybrid circuits $281.1 \mathrm{M} \$$

TOTAL $455.3 \mathrm{M} \$$

It can be seen that there is reasonable agreement between the two estimates. However, it should be noted that the figure for non wire wound potentiometers will include those made using moulded carbon and metal foil tracks.

\section{EUROPEAN SITUATION}

Figures given by the Panel for 1976 and estimates for 1977 and 1978 are shown in Table I. Table I also includes estimates of the percentage of the markets in each country associated with internal (i.e. captive) use. Figures previously given for the U.S. are also included, together with estimates of the commercial market in Japan.

No comparable figures are available from other sources, Electronics ${ }^{3}$ only giving classifications such as "Hybrid I.C.'s all types" and no classification on "Networks".

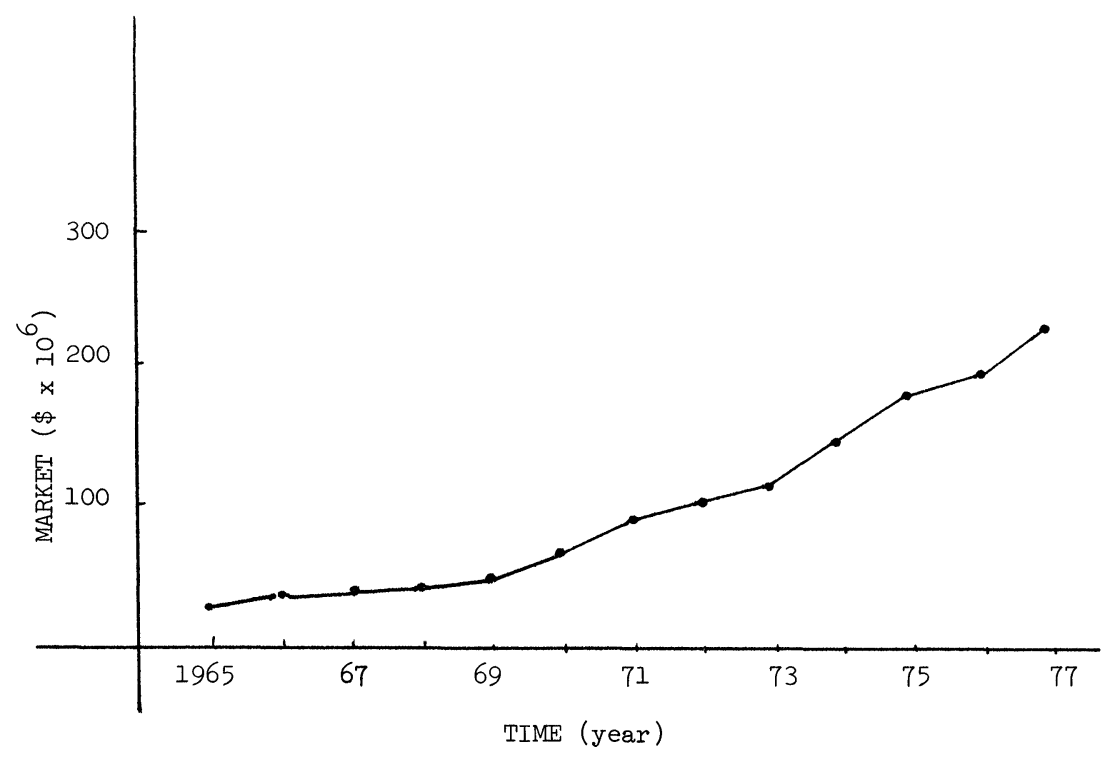

FIGURE 2 Growth of multichip hybrid circuit production for the non-captive market in the U.S. (after Waite, Ref. 1). 
TABLE I

Total markets in Europe for thin and thick film networks and multichip hybrids

\begin{tabular}{|c|c|c|c|c|c|c|}
\hline & \multicolumn{2}{|l|}{1976} & \multicolumn{2}{|l|}{1977} & \multicolumn{2}{|l|}{1978} \\
\hline & $\begin{array}{l}\text { Total } \\
\text { Market } \\
\text { (\$M) }\end{array}$ & $\%$ Captive & $\begin{array}{l}\text { Total } \\
\text { Market } \\
\text { (\$M) }\end{array}$ & $\%$ Captive & $\begin{array}{l}\text { Total } \\
\text { Market } \\
\text { (\$M) }\end{array}$ & $\%$ Captive \\
\hline Japan & $57^{\mathrm{a}}$ & - & $65^{\mathrm{a}}$ & - & - & - \\
\hline U.S.A. & 1000 & 75 & 1200 & 75 & - & 60 \\
\hline Germany & 30 & 70 & 35 & 75 & 50 & 80 \\
\hline U.K. & 30 & 55 & 40 & 55 & 45 & 55 \\
\hline France $^{c}$ & 40 & 70 & 50 & 75 & 56 & 70 \\
\hline Italy ${ }^{b}$ & 8 & 50 & 15 & 30 & 20 & 20 \\
\hline Scandinavia and Finland & 24 & 50 & 32 & 60 & 40 & 60 \\
\hline Benelux & 9 & 60 & 11 & 65 & 13 & 65 \\
\hline $\begin{array}{l}\text { Others, including Switzerland, } \\
\text { Spain and Portugal }\end{array}$ & 12 & - & 15 & - & 18 & - \\
\hline Total European & 153 & - & 198 & - & 242 & - \\
\hline
\end{tabular}

\footnotetext{
${ }^{a}$ Commercial production only.

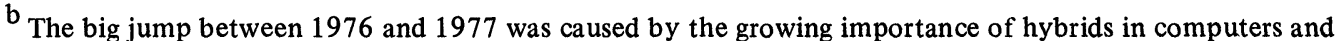
automation. Telecommunications accounts for half of the output in 1976 but only $20 \%$ of the output in 1977.

${ }^{c}$ This figure excludes I.B.M. captive market production.
}

The percentage of markets associated with thin film circuits was also commented upon by the Panel. The percentages suggested were as shown in Table II.

TABLE II

Percentage of total hybrid market associated with thin film circuits

\begin{tabular}{ll}
\hline U.S.A. & $5 \%-10 \%$ \\
Germany & $15 \%-20 \%$ \\
U.K. & $10 \%$ \\
France $^{\mathrm{a}}$ & $80 \%$ \\
Italy $^{\text {Nordic }}{ }^{\mathrm{b}}$ & $20 \%$ \\
Nord & $15 \%$ \\
\hline
\end{tabular}

a A high percentage of thin film circuits shown in the Table for France are associated with tantalum circuit technology used in telecommunications.

$\mathrm{b}_{\text {These figures disguise a wide spread in thin film }}$ circuits production in the three Scandinavian countries:90\% Norway, $14 \%$ Sweden, $25 \%$ Denmark. These have been summed and given a weighted average.

The Panel was also asked to give its comments with regard to the percentage of the European markets associated with hermetic packaging. Not all members were able to give figures in this area, but Table III shows the figures which were made available.
TABLE III

Percentage of market, in cash terms, associated with hermetically packaged circuits

\begin{tabular}{ll}
\hline U.S.A. & $85 \%$ \\
Germany & $65 \%$ \\
U.K. & $20 \%$ \\
France & $90 \%$ \\
\hline
\end{tabular}

\section{DISCUSSION}

The forecast for European business expansion for hybrid circuits shows approximately a $29 \%$ increase over the period 1976/77. This figure, if achieved, will be greater than the predictions made for monolithic integrated circuits ${ }^{3}(24 \%)$ and will also have the great advantage that unlike supplies of semiconductors, most of it will be met by indigenous European manufacturers.

At $1000 \mathrm{M} \$$ for 1976 and $1200 \mathrm{M} \$$ for 1977 , the large size of hybrid circuit activity in the U.S.A. has surprised many people. The total non-captive integrated circuit business for 1977 is predicted as $1927.3 \mathrm{MS}^{3}$ In the case of integrated circuit production, the majority of the output is of a non-captive nature in direct contrast to the situation previously 
identified in Table I for hybrid systems. If one therefore adds to the non-captive predicted figure, a further approximately $1000 \mathrm{M} \$$ for captive work, this would give, in round terms, a total integrated circuit business, both captive and non-captive for 1977, of $3000 \mathrm{M} \$$. If one adds to this the $1200 \mathrm{M} \$$ for the hybrid activity, this makes a total microelectronic business of approximately $4000 \mathrm{M} \$$ and thus it can be seen that the hybrid activity represents about $25 \%$ of this total business.

There appears to be a notable lack of understanding amongst European governments as to the future importance of hybrid technology. Hybrid Technology already provides production based on large quantities of a limited range of standard circuits in the fields of automotive, electronics, telecommunications, radio and television. Hybrid circuit technology is also developing into a basic and economic method of assembling electronic equipment.

To concentrate only on the high volume production aspects of integrated circuit technology ignores what is happening to the mechanics of electronic equipment design and construction. A new era is emerging in which computers allow increasing complexity and consequently lower quantities to be handled in design and manufacturing stages at acceptable cost. These statements apply equally to the semiconductor integrated circuit industry, whose sales matrix also contains a rising proportion of complex circuits made in far lower quantities than hitherto.

In this role hybrid circuits should be considered alongside conventional epoxy printed circuits, i.e. they are benefiting the industry by proliferating in wide variety, comparatively small batch runs with extended circuit complexity. Recent trends in U.S. and U.K. military, telecommunication and medical hybrids support this view.

The trend towards increasing complexity will affect many commercial interfaces in the electronics industry. Some integrated circuit manufacturers who now see themselves as purveyors of high technology products to all, will through acquisition of added value and application knowledge, become orientated towards one particular product sector. Many will develop into specialist equipment firms.

Equipment makers should not view this with alarm. Increasing the component supplier's application knowledge and product complexity range allows the systems house to concentrate on its major role - as designer and assembler of equipment, having progressively greater sophistication and capability.

Conversely, as processes and equipment become better defined, more big equipment manufacturing companies will risk investment in a combined film and semiconductor circuit capability. They will then be accepting heavy ongoing financial commitments to keep abreast in the technology race.

Alternatively and at less risk, they can utilise the new trade interface being created by rectilinear chiphybrid technology. This means buying patterned substrates and chips; assembling, encapsulating and testing themselves. Obviously, the added value held by the component manufacturer is then reduced.

From such premises one can postulate a balanced figure situation in which the component/equipment interface becomes steadily less well defined, but does not change radically the present organisation of the electronics industry.

\section{CONCLUSIONS}

This paper summarizes the discussions held on the Economics of Hybrid Circuit Technology at the First European Conference on Hybrid Microelectronics. The assumptions used in arriving at the captive and non-captive markets for hybrid systems, both in the U.S.A. and in Europe, have been discussed, and the size of the business in both areas has been identified. It has been noted that one of the strongest reasons for the existence of hybrid microelectronics is the facility of interconnections which the hybrid technique offers and therefore the growth of hybrids will be closely associated with the growth of large-scale integration. This method of interconnection, however, will not replace printed circuit board techniques as the two facilities offer complementary interconnection possibilities.

Reliability must be the keynote of future advancement and this will only arise from standardisation.

\section{ACKNOWLEDGEMENTS}

The authors have produced this paper as a report on the discussions held at the First European Conference on Hybrid Microelectronics, on the Economics of Hybrid Circuit Technology. They are therefore totally indebted to all their colleagues who have made comments, particularly to the members of the Discussion Panel and with special reference to Messrs. Kirby and Waite.

\section{REFERENCES}

1. P. L. Kirby, Private Communication, 1977.

2. G. Waite, Proc. European Hybrid Microelectronics Conf., pp. 28-1 to 28-8, 2-4 May, 1977.

3. "World Electronics Markets," Electronics, 50, pp. 81-104, Jan. 6, 1977. 

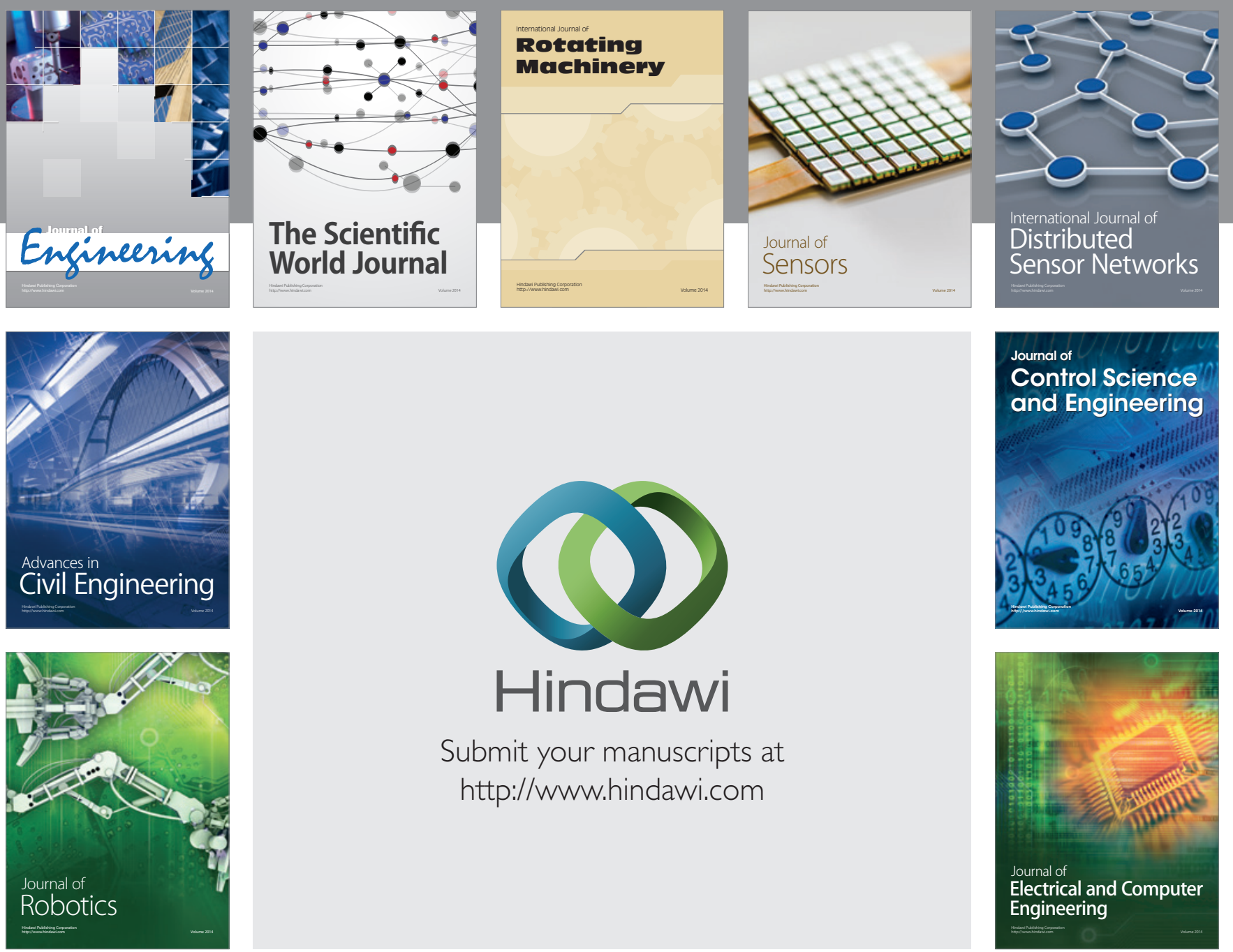

Submit your manuscripts at

http://www.hindawi.com
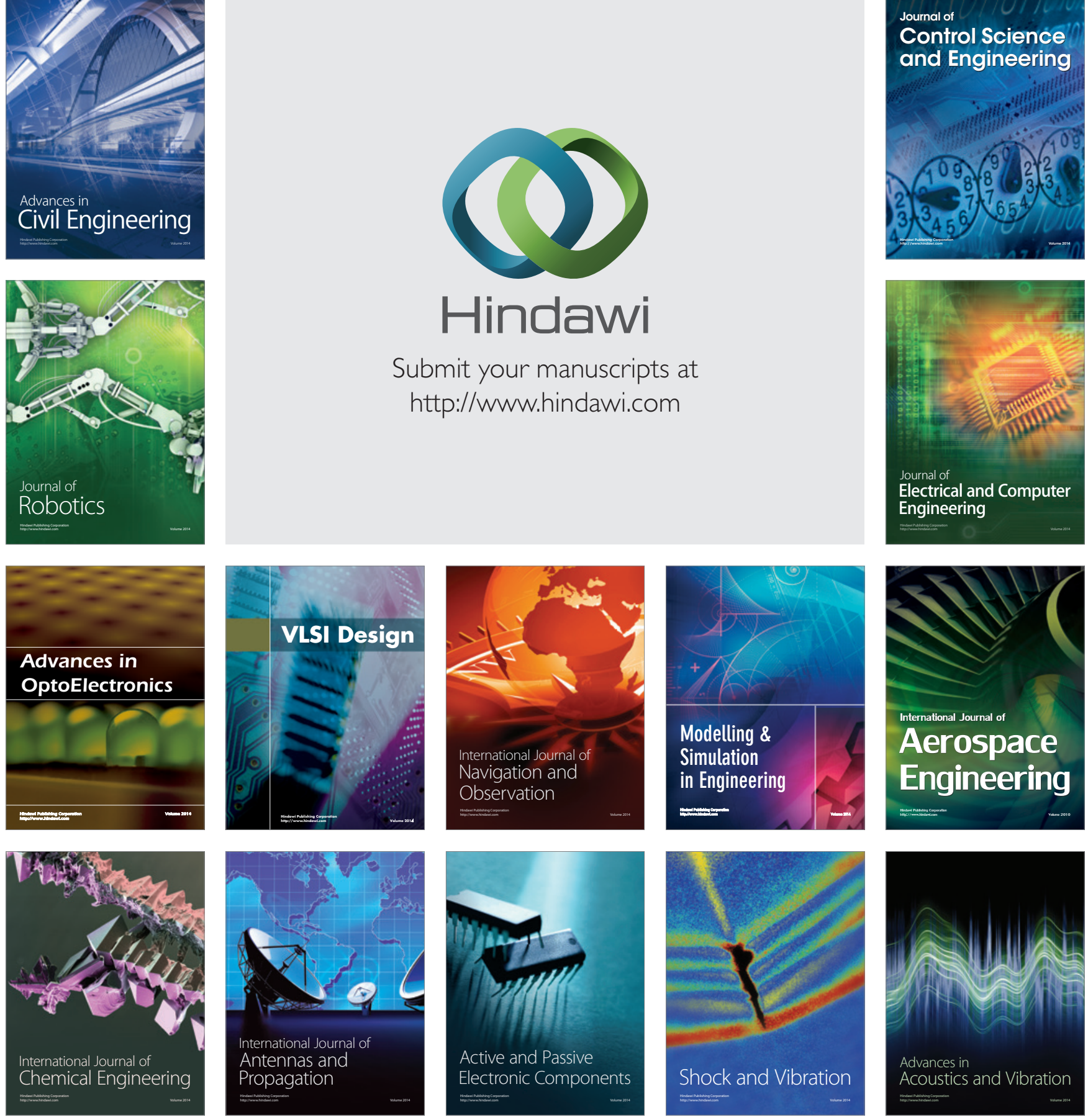